exploited. Another good direction would be smooth dynamics, where the recent [1] would be useful.

Parry has managed to compress a stimulating cross-section of ergodic theory into a book of relatively modest size. Those who study it will be amply rewarded.

\title{
REFERENCES
}

[1] Michael Irwin. Smooth Dynamical Systems. Academic Press: New York, 1980.

[2] Donald S. Ornstein. Ergodic Theory, Randomness, and Dynamical Systems. Yale Univ. Press: New Haven, 1974.

[3] Paul Shields. The Theory of Bernoulli Shifts. Univ. of Chicago Press: Chicago, 1973.

[4] Ya. G. Sinai. Introduction to Ergodic Theory. Princeton Univ. Press: Princeton, 1977.

D. LIND

\section{RECURRENCE IN ERGODIC THEORY AND COMBINATORIAL NUMBER THEORY}

By H. FURSTENBERG: pp. 199. Princeton University Press: Princeton, New Yersey, 1981

Ergodic theorists will be aware of the author's long-standing interest in the application of dynamics and probability theory to the solution of number theoretical problems. Already in his Annals study 'Stationary processes and prediction theory' [1] and in his 'skew products' paper [2] there appears a proof of Weyl's theorem on the uniform distribution of polynomial functions of a natural number, and in his 'Disjointness' paper [4] he obtains fascinating results concerning semi-groups of endomorphisms of a torus.

Here we find the most convincing testimony for the power of dynamical techniques in combinatorial number theory. The book under review is devoted to ergodic theoretic and topological dynamical proofs of the following well known theorems:

VAN DER WAERDEN. (V.d.W)

If $\mathbb{Z}$ is partitioned into sets $B_{1}, B_{2}, \ldots, B_{q}$, then one of the $B_{j}$ contains arithmetic progressions of arbitrary length.

Hindman. (H)

If $\mathbb{N}$ is partitioned into $B_{1}, \ldots, B_{q}$, then one of the $B_{j}$ has the property that there exist $x_{1} \leq x_{2} \leq \cdots$ such that all the elements $x_{i}$ together with $x_{i_{1}}+\cdots+x_{i_{k}},\left(i_{1}<i_{2} \cdots<i_{k}\right)$, belong to $B_{j}$.

RADO. (R)

This is more complicated to state, but it gives necessary and sufficient conditions on a system of equations

$$
\sum a_{i j} x_{j}=0
$$

to have a solution in a single element of an arbitrary finite partition of $\mathbb{N}$. 
SZEMEREDI. (Sz)

If $B \subset \mathbb{Z}$ has positive upper Banach density (i.e. there exists $b_{n}-a_{n} \rightarrow \infty$ such that

card $B \cap\left[a_{n}, b_{n}\right] / b_{n}-a_{n} \geq d>0$

for all $n$ ), then $B$ contains arithmetic progressions of arbitrary length.

The book is divided into three parts. The first is devoted to (V.d.W) and related theorems and uses the techniques of topological dynamics, as does the third which is devoted to $(\mathrm{H})$ and $(\mathrm{R})$. The second part is concerned with ergodic theory and the proof of $(\mathbf{S z})$.

The basic observation is that a partition $\left(B_{1}, \ldots, B_{q}\right)$ can be identified with a sequence $x=\left\{x_{n}\right\}$ of digits chosen from the finite alphabet $1, \ldots, q$ where $x_{n}=k$ if and only if $n \in B_{k}$. Thus a partition $\left(B_{1}, \ldots, B_{q}\right)$ of $\mathbb{N}$ corresponds to a point in

$$
X^{+}=\prod_{n=0}^{\infty}\{1, \ldots, q\}
$$

and a partition of $\mathbb{Z}$ corresponds to a point in

$$
X=\prod_{n=-\infty}^{\infty}\{1, \ldots, q\} .
$$

A well known theorem of Birkhoff states that if $T$ is a homeomorphism of a compact metric space $Y$ then there exists a $T$ recurrent point $y$ in $Y$ :

there exists $m_{n} \rightarrow \infty$ such that $T^{m_{n}} y \rightarrow y$.

Birkhoff's theorem is generalized in Part $I$ to $k$ commuting maps $T_{1}, \ldots, T_{k}$. A point $y$ is called multiply recurrent with respect to these maps if there exists $m_{n} \rightarrow \infty$ such that

$$
T_{1}^{m_{n}} y \rightarrow y, \ldots, T_{k}^{m_{n}} y \rightarrow y .
$$

The Birkhoff multiple recurrence theorem (B.M.R) states that a multiply recurrent point always exists. Specialising to the space $Y$, the orbit closure under the shift of the given partition sequence $x$, and considering the maps $T, T^{2}, \ldots, T^{k}$, where $T$ is the restriction of the shift to $Y$, the conclusion is that there exists $y \in Y$ and $m_{n} \rightarrow \infty$ such that

$$
T^{m_{n}} y \rightarrow y, \ldots, T^{k m_{n}} y \rightarrow y .
$$

However, $y$ is arbitrarily close to some $T^{l} x$ so that we have for all $\varepsilon>0$ and some $l$,

$$
d\left(T^{m_{n}+l} x, T^{l} x\right)<\varepsilon, \ldots, d\left(T^{k m_{n}+l} x, T^{l} x\right)<\varepsilon
$$

In particular

$$
x_{m_{n}+l}=x_{l}, \ldots, x_{m_{n} k+l}=x_{l}
$$

so that for some $j$,

$$
m_{n}+l \in B_{j}, \ldots, m_{n} k+l \in B_{j}
$$

i.e. $B_{i}$ contains an arithmetic progression of length $k$. The (B.M.R) theorem is also used to establish a multiple dimensional generalization of (V.d.V) (for partitions of $\mathbb{Z}^{n}$ or $\mathbb{N}^{n}, n>1$ ) due to Grünwald. 
Perhaps the central result of the book is the ergodic theoretic proof of Szemeredi's theorem. The theorem itself was conjectured by Erdös and Turán in the 1930's. In 1952 Roth proved that the subset $B \subset \mathbb{N}$ (of positive upper density) contains an arithmetic progression of length 3 . Then, in 1969, Szemeredi showed that A.P.'s of length 4 existed and in 1975 he established the conjecture completely.

Furstenberg's proof is inspired by the same philosophy as his proof of (V.d.W) but with the 'positive upper density' condition the appropriate tool is ergodic theory. For with this condition it is easy to establish the existence of a shift invariant probability $m$ on $Y$, the orbit closure of

$$
x=\chi_{B} \in \prod_{n=0}^{\infty}\{0,1\} .
$$

(Moreover $m\left\{y: y_{0}=1\right\}>0$.) So the appropriate theorem, which implies Szemeredi's theorem, is (with $T_{1}=T, \ldots, T_{k}=T^{k}$, for $T$ the shift on $Y$ ) the multiple Poincaré recurrence theorem (M.P.R):

If $T_{1}, \ldots, T_{k}$ are commuting m.p.t. of the probability space $(X, \mathscr{B}, m)$ then for $m(A)>0$ there exists $n$ such that

$$
m\left(A \cap T_{1}^{-n} A \cap \cdots \cap T_{k}^{-n} A\right)>0 .
$$

The proof of (M.P.R) is rather more sophisticated than the proof of (B.M.R) Although measure-theoretic, it is nevertheless motivated by Furstenberg's 'Distal' paper [3]. In this paper he provided a structure theorem for distal flows and showed how such flows can be obtained as a transfinite inverse limit of isometric extensions starting from the trivial flow. Furstenberg's first proof of $(\mathrm{Sz})$ leaned on Zimmer's proof of the measure-theoretic analogue of the structure theorem. The approach in this book is via a very general structure theorem for measure-preserving (not necessarily ergodic) transformations, and involves the concepts of relative weakmixing, isometric (compact) extension and primitive extension. Suppose a finitely generated abelian group $\Gamma$ of measure-preserving transformations acts on both $(X, \mathscr{B}, m)$ and $\left(X^{\prime}, \mathscr{B}^{\prime}, m^{\prime}\right)$ and suppose there is a homomorphism $\alpha: X \rightarrow X^{\prime}$ such that $\gamma \alpha=\alpha \gamma$. Then $(X, \mathscr{B}, m, \Gamma)$ is said to be a primitive extension of $\left(X^{\prime}, \mathscr{B}{ }^{\prime}, m^{\prime}, \Gamma\right)$ if

$$
\Gamma=\Gamma_{1} \times \Gamma_{2}
$$

and $\left(X, \mathscr{B}, m, \Gamma_{1}\right)$ is an isometric extension of $\left(X^{\prime}, \mathscr{B}^{\prime}, m^{\prime}, \Gamma_{1}\right)$ whereas $\left(X, \mathscr{B}, m, \Gamma_{2}\right)$ is a relatively w.m. extension of $\left(X^{\prime}, \mathscr{B}^{\prime}, m^{\prime}, \Gamma_{2}\right)$. The structure theorem reads as follows:

If $\Gamma$ is a finitely generated abelian group of measure-preserving transformations of the Lebesgue space $(X, \mathscr{B}, m)$ then there is an ordinal $\eta$ and a collection of factors

$$
\left(X_{\zeta}, \mathscr{B}_{\zeta}, m_{\zeta}, \Gamma\right) \text {, }
$$

one for each $\zeta \leq \eta$, such that $X_{0}$ is a point (trivial system),

$$
\left(X_{\eta}, \mathscr{B}_{\eta}, m_{\eta}\right)=(X, \mathscr{B}, m)
$$

and for each $\zeta<\boldsymbol{\eta}$,

$$
\left(\boldsymbol{X}_{\zeta+1}, \mathscr{B}_{\zeta+1}, m_{\zeta+1}, \Gamma\right)
$$


is a primitive extension of $\left(X_{\zeta}, \mathscr{B}_{\zeta}, m_{\zeta}, \Gamma\right)$, and for each limit ordinal $\zeta \leq \eta$,

$$
\left(X_{\zeta}, \mathscr{B}_{\zeta}, m_{\zeta}, \Gamma\right)
$$

is the inverse limit of systems with lower ordinal.

Using this theorem and results to the effect that the (M.P.R) theorem persists under isometric or relative weak-mixing extensions, the author achieves the (M.P.R) theorem in the form:

If $T_{1}, T_{2}, \ldots, T_{k}$ are commuting m.p.t. of $(X, \mathscr{B}, m)$ then for $m(A)>0$ we have

$$
\underline{\lim } \frac{1}{N} \sum_{n=0}^{N-1} m\left(T_{1}^{-n} A \cap T_{2}^{-n} A \cdots \cap T_{k}^{-n} A\right)>0 .
$$

One advantage of Furstenberg's approach to $(\mathrm{Sz})$ is that it yields a multi-dimensional version of the theorem at no extra cost. A disadvantage, so far, is that it yields no bounds in the finite version of the theorem, unlike Szemeredi's proof.

It seems to be the author's intention that readers should prepare themselves for the long march by first understanding the two simplest cases of the theorem namely the (M.P.R) theorem for isometric actions of $\mathbb{Z}^{\nu}$ and w.m. actions of $\mathbb{Z}^{\nu}$, and the reviewer would concur with this advice. The author also isolates the special case of Szemeredi's theorem proved by Roth for an independent and more direct proof employing ergodic techniques, $\S 4$ Chapter 4 . The reviewer found some aspects of this section confusing and could not decide, for a long time, whether $L^{\infty}(X, \mathscr{B}, m)$ was to be interpreted as the usual Banach space or whether it meant the space of all bounded measurable functions - probably either will do - but the notation $\mathbf{X}$, bold type, when appearing in $L^{\infty}(\mathbf{X}) \otimes L^{\infty}(\mathbf{X})$, but not in $L^{\infty}(X, \mathscr{B}, m)$, confused. Moreover it was not immediately obvious how prop. 4.20 followed from 4.19 since the former requires ergodicity and the latter does not - presumably one appeals to ergodic decomposition, but this concept appears only later.

The third section is topological dynamical and to give some idea of the author's aim we restrict attention to his proof of Hindman's theorem, which is largely based on a theorem of Auslander and Ellis and which reads:

In a dynamical system on a compact metric space every point is proximal to a uniformly recurrent point in its orbit closure.

An I.P. set is a subset of $\mathbb{N}$ which consists of a sequence $x_{1}, x_{2}, \ldots$ together with all sums

$$
x_{i_{1}}+\cdots+x_{i_{k}}
$$

with $i_{1}<i_{2}<\cdots<i_{k}$. A central set $S \subset \mathbb{N}$ is one for which there exists a homeomorphism $T$ of a compact metric space $X$ and

$$
S=\left\{n: T^{n} x \in U_{y}\right\}
$$

where $x \in X, y$ is uniformly recurrent and proximal to $x$, and $U_{y}$ is a neighbourhood of $y$. Hindman's theorem is approached as follows:

If $B_{1}, \ldots, B_{q}$ is a partition of $\mathbb{N}$ then one of the $B_{j}$ contains a central set.

Central sets contain arbitrarily long arithmetic progressions. Central sets contain I.P. sets. Thus (Hindman's theorem) one of the $\boldsymbol{B}_{\boldsymbol{j}}$ contains an I.P. set. 
Like the rest of the book this section is rich in new ideas. The author has provided ergodic theorists and combinatorial number theorists with a variety of techniques and a sound foundation for an area of research which promises to be especially fruitful. In the process he has also revitalised abstract ergodic theory.

\section{REFERENCES}

[1] H. Furstenberg. Stationary Processes and Prediction Theory. Princeton Univ. Press: Princeton, 1960.

[2] H. Furstenberg. Strict ergodicity and transformations of the torus. Amer. J. Math. 83 (1961), 573-601.

[3] H. Furstenberg. The structure of distal flows. Amer. J. Math. 85 (1963), 477-515.

[4] H. Furstenberg. Disjointness in ergodic theory, minimal sets, and a problem in diophantine approximation. Math. Syst. Th. Vol. 1. No. 1 (1967), 1-49.

W. PARRY 